\title{
Eye examination is important in patients with tubulointerstitial nephritis
}

\author{
Timo Jahnukainen • Marja Ala-Houhala • \\ Ville Saarela $\cdot$ Matti Nuutinen
}

Received: 11 February 2011 /Accepted: 21 February 2011/Published online: 16 April 2011

(C) IPNA 2011

Sirs,

Dr. Lava and his colleagues present the interesting case of a patient with idiopathic tubulointerstitial nephritis (TIN) and late-onset uveitis [1]. The authors highlight the importance of ophthalmologic follow-up in TIN patients, and their case demonstrates that uveitis may develop in a patient even during ongoing corticosteroid therapy. In our study [2], uveitis was diagnosed in five patients between 3 and 15 months after onset of nephritis, i.e., in $42 \%$ of the patients

T. Jahnukainen $(\triangle)$

Department of Pediatric Nephrology and Transplantation, Children's Hospital, University of Helsinki and Helsinki University Central Hospital,

Stenbäckinkatu 11, P.O. Box 281, 00029 Helsinki, Finland

e-mail: timo.jahnukainen@hus.fi

\section{Ala-Houhala}

Department of Pediatrics, Tampere University Hospital,

Tampere, Finland

V. Saarela

Department of Ophthalmology, Oulu University Hospital, Oulu, Finland

\section{Nuutinen}

Department of Pediatrics and Adolescence,

Oulu University Hospital,

Oulu, Finland with TIN and uveitis. Two of these patients were not receiving any medication at the time uveitis was diagnosed, three patients were on corticosteroids $(0.15-0.65 \mathrm{mg} / \mathrm{kg} /$ day $)$, with one also on cyclosporin A.

The case report by Dr. Lava et al. as well as our own experience suggest that uveitis may develop in TIN patients during the weaning of systemic corticosteroid treatment. Uveitis may be asymptomatic and, therefore, ophthalmologic slit lamp examination should be performed before the corticosteroid dose is reduced. If necessary, topical treatment should be started in order to avoid long-term eye complications. The ophthalmological follow-up of both TIN and TINU patients for at least 12 months is motivated by three factors: (1) uveitis may develop late and be asymptomatic; (2) uveitis may relapse after apparent healing; (3) uveitis may turn out to be chronic.

\section{References}

1. Lava SAG, Bucher O, Simonetti GD, Tchumi S (2011) Development of uveitis during systemic corticosteroid therapy in TINU syndrome. Pediatr Nephrol doi:10.1007/s00467-011-1853-6

2. Jahnukainen T, Ala-Houhala M, Karikoski R, Kataja J, Saarela V, Nuutinen M (2011) Clinical outcome and occurrence of uveitis in children with idiopathic tubulointerstitial nephritis. Pediatr Nephrol 26:291-299 